\title{
PERIODIC ORBITS OF THE PLANAR COLLISION RESTRICTED 3-BODY PROBLEM
}

\author{
JAUME LLIBRE ${ }^{1}$ AND DANIEL PAŞCA ${ }^{2,3}$
}

\begin{abstract}
Using the continuation method we prove that the circular and the elliptic symmetric periodic orbits of the planar rotating Kepler problem can be continued into periodic orbits of the planar collision restricted 3-body problem. Additionally, we also continue to this restricted problem the so called "comets orbits".
\end{abstract}

\section{INTRODUCTION}

We consider a special case of the restricted 3-body problem, called collision restricted 3-body problem. In our problem the two primaries with equal masses are moving in a collision orbit of the 2-body problem, and the infinitesimal mass is moving on a plane orthogonal to the line of motion of the primaries. The purpose of this paper is to find symmetric periodic orbits of this problem using the continuation method developed by Poincaré which is one of the most frequently used methods for proving the existence of periodic orbits. Starting with Poincaré in [8], which used this method to prove the existence of periodic orbits for circular planar restricted 3-body problem, see [10], this method was used also by other authors in different problems like spatial isosceles 3-body problem (see [1], [5]), circular and elliptic Sitnikov problem (see [3], [4]), collinear restricted 3-body problem (see [2]), etc. In [6], we find a good discussion on the applicability of the Poincaré's continuation method to different $N$-body problems.

We give the equations of motion of the collision restricted 3-body problem in Section 2. In Section 4 we introduce three different kinds of symmetric periodic orbits. In section 3,5 and 6 we will prove the continuation of the circular orbits (see Theorem 1), of the different three kinds of symmetric

1991 Mathematics Subject Classification. Primary 70F15(70F07).

Key words and phrases. Collision restricted 3-body problem, continuation method, periodic orbits.

$\dagger$ The first author is partially supported by a DGICYT grant number BFM 200204236-C02-02 and by a CICYT grant number 2001SGR00173. The second author was supported by Ministerio de Educacioń y Ciencia, grant number SB2003-0337. 
elliptic orbits (see Theorems 5, 6, 7) and of the comets orbits (see Theorem 8) from the rotating Kepler problem to the collision restricted 3-body problem.

\section{The COLLISION RESTRICTED 3-BODY PROBLEM}

Let $m_{1}=m_{2}$ be two punctual masses (called primaries) describing a collision orbit of the 2 -body problem. We consider an infinitesimal mass $m_{3}$ that moves on the plane $\Pi$ orthogonal to the line of motion of the primaries through their center of mass. We choose the units of mass, length and time so that $m_{1}=m_{2}=1 / 2$, the gravitational constant $G=1$, and the period of the two primaries is $2 \pi$. We take the origin of coordinates at the center of mass, the plane of the motion of the infinitesimal body is the $\left(X_{1}, X_{2}\right)$-plane and the motion of the primaries take place on the $z$-axis (see Figure 1). Then the equations of motion of the infinitesimal mass are

$$
\begin{aligned}
& \ddot{X}_{1}=-\frac{X_{1}}{\left(X_{1}^{2}+X_{2}^{2}+z^{2}(t)\right)^{3 / 2}}, \\
& \ddot{X}_{2}=-\frac{X_{2}}{\left(X_{1}^{2}+X_{2}^{2}+z^{2}(t)\right)^{3 / 2}},
\end{aligned}
$$

where $z(t)$ is the solution of the elliptic collision 2-body problem, which we take as

$$
z(t)=\mu(1-\cos E(t))
$$

where $\mu>0$ is a small parameter and $E(t)$ is the eccentric anomaly which is a function of time via Kepler's equation

$$
E-\sin E=t
$$

We note that since $E$ is $2 \pi$-periodic, $z$ will be $2 \pi$-periodic. For more details on the motion of the primaries see [9].

We want to analyze the continuation of periodic solutions for this problem from $\mu=0$ to $\mu>0$ small. Like in the case of planar circular restricted 3 -body problem, we cannot prove the continuation of the periodic solutions in fixed coordinates because the system in these coordinates is very degenerated. For this reason, we consider a rotating coordinate system $\left(x_{1}, x_{2}, z\right)$ related with the fixed one through the following formula

$$
\left(\begin{array}{c}
x_{1} \\
x_{2} \\
z
\end{array}\right)=\left(\begin{array}{ccc}
\cos t & \sin t & 0 \\
-\sin t & \cos t & 0 \\
0 & 0 & 1
\end{array}\right)\left(\begin{array}{c}
X_{1} \\
X_{2} \\
z
\end{array}\right) .
$$




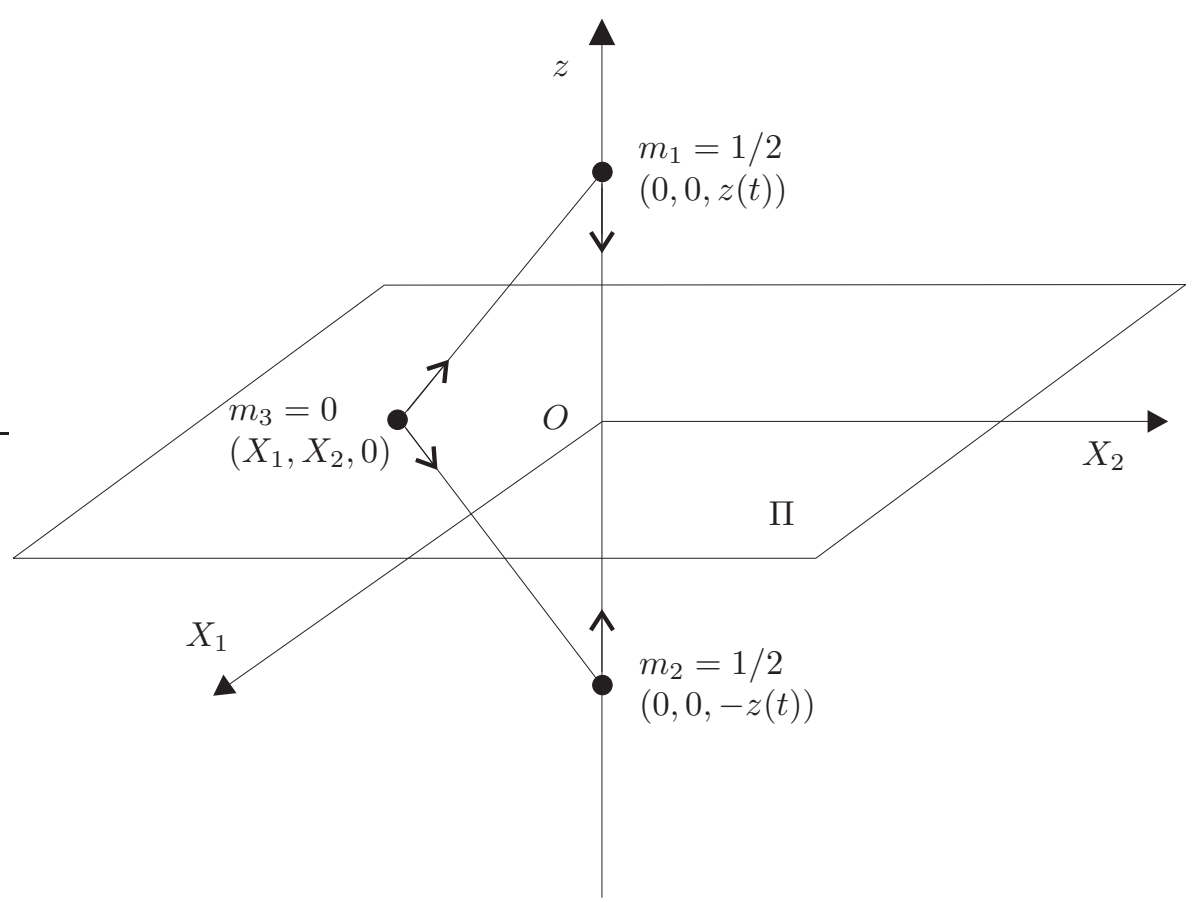

Figure 1. The elliptic collision 2-dimensional restricted 3-body problem

The Hamiltonian governing the motion of the infinitesimal mass for this collision restricted 3-body problem is

$$
H=\frac{\|y\|^{2}}{2}-x^{T} K y-\frac{1}{\left[\|x\|^{2}+z^{2}(t)\right]^{1 / 2}},
$$

where $x=\left(x_{1}, x_{2}\right)$ and $y=\left(y_{1}, y_{2}\right)$ are conjugate, $K=\left(\begin{array}{cc}0 & 1 \\ -1 & 0\end{array}\right)$ and $x^{T} K y$ is just the angular momentum. Since $\mu$ is a small parameter the Hamiltonian can be written into the form

$$
H=\frac{\|y\|^{2}}{2}-x^{T} K y-\frac{1}{\|x\|}+O\left(\mu^{2}\right) .
$$

When $\mu=0, H$ is the Hamiltonian of the 2-body problem, or Kepler problem, in rotating coordinates. There are two main differences between the Hamiltonian given by (2) and the corresponding Hamiltonian in the 
planar circular restricted 3-body problem. The first one is that for the collision restricted 3 -body problem the perturbation is of the order $\mu^{2}$ instead of the order $\mu$ for the planar circular restricted 3 -body problem. The second is that our Hamiltonian (2) depends explicitly on the time (i.e. it is non-autonomous) but is $2 \pi$-periodic. The dependence is contained in the terms of $O\left(\mu^{2}\right)$.

\section{The CONTINUATion OF THE CiRCUlar ORBits}

First we change the coordinates from the cartesian coordinates $\left(x_{1}, x_{2}, y_{1}, y_{2}\right)$ to the polar coordinates $(r, \theta, R, \Theta)$. This change of coordinates is given by

$$
\begin{array}{cc}
x_{1}=r \cos \theta, & x_{2}=r \sin \theta \\
y_{1}=R \cos \theta-\frac{\Theta}{r} \sin \theta, & y_{2}=R \sin \theta+\frac{\Theta}{r} \cos \theta .
\end{array}
$$

For more details see [7]. In these new coordinates the Hamiltonian is of the form

$$
H=\frac{1}{2}\left(R^{2}+\frac{\Theta^{2}}{r^{2}}\right)-\Theta-\frac{1}{r}+O\left(\mu^{2}\right) .
$$

Now, following Section C of Chapter VI of [7], we can prove in the same way the continuation of the circular orbits (or periodic orbits of the first kind in the terminology introduced by Poincaré; for more details see [10]) from $\mu=0$ to $\mu>0$ sufficiently small.

Theorem 1. Let $p, q$ relatively prime integers and $T=2 \pi p / q$. Then the circular $T$-periodic orbits of the Kepler problem in rotating coordinates with angular momentum $c$ can be continued to a periodic orbit of period $2 \pi p$ of the collision restricted 3-body problem for small values of $\mu>0$.

Proof. For $\mu=0$ the equations of motion are

$$
\begin{gathered}
\dot{r}=R, \quad \dot{R}=\frac{\Theta^{2}}{r^{3}}-\frac{1}{r^{2}}, \\
\dot{\theta}=\frac{\Theta}{r^{2}}-1, \quad \dot{\Theta}=0 .
\end{gathered}
$$

Let $\Theta=c$ be a fixed constant. The circular orbit $R=0, r=c^{2}$ is a periodic solution with period $\left|2 \pi c^{3} /\left(1-c^{3}\right)\right|$ (this is the time for $\theta$ to increase by $2 \pi)$. We wish to consider the case when

$$
\left|2 \pi c^{3} /\left(1-c^{3}\right)\right|=\frac{2 \pi p}{q},
$$


which implies that

$$
c=\left(\frac{p}{p+q}\right)^{1 / 3} \text { or } c=\left(\frac{p}{p-q}\right)^{1 / 3} .
$$

Linearizing the $r$ and $R$ equations about this solution gives

$$
\dot{r}=R, \quad \dot{R}=-c^{-6} r,
$$

which has solutions of the form $\exp \left( \pm i t / c^{3}\right)$, and so the nontrivial multipliers of the circular orbits are $\exp \left( \pm i 2 \pi /\left(1-c^{3}\right)\right)$ which are not +1 , since $1 /\left(1-c^{3}\right)$ is not an integer. We apply the results of the Appendix to the Hamiltonian system defined by (3) thinking this system as a $2 \pi p$-periodic system, and continuing the circular orbit of period $2 \pi p / q$ rotated $q$ times. Then, this orbit can be continued for $\mu>0$ small.

\section{Symmetric Periodic orbits}

The equations of motion of the infinitesimal mass in the rotating coordinates are

$$
\begin{aligned}
& \ddot{x}_{1}=x_{1}+2 \dot{x}_{2}-\frac{x_{1}}{\left(x_{1}^{2}+x_{2}^{2}+z^{2}(t)\right)^{3 / 2}}, \\
& \ddot{x}_{2}=x_{2}-2 \dot{x}_{1}-\frac{x_{2}}{\left(x_{1}^{2}+x_{2}^{2}+z^{2}(t)\right)^{3 / 2}} .
\end{aligned}
$$

It is easy to check that the equations of motion (4) are invariant under the symmetry

$$
\left(t, x_{1}, \dot{x}_{1}, x_{2}, \dot{x}_{2}\right) \longrightarrow\left(-t, x_{1},-\dot{x}_{1},-x_{2}, \dot{x}_{2}\right) .
$$

This means that if $\varphi(t)=\left(x_{1}(t), \dot{x}_{1}(t), x_{2}(t), \dot{x}_{2}(t)\right)$ is a solution of system (4), then also $\psi(t)=\left(x_{1}(-t),-\dot{x}_{1}(-t),-x_{2}(-t), \dot{x}_{2}(-t)\right)$ is a solution. We note that this symmetry corresponds to a symmetry with respect to the $x_{1}$-axis, and will be denoted by $S_{1}$. In fact, is well known the following result.

Proposition 2. Let $\varphi(t)=\left(x_{1}(t), \dot{x}_{1}(t), x_{2}(t), \dot{x}_{2}(t)\right)$ be a solution of system (4). If $\dot{x}_{1}(t)$ and $x_{2}(t)$ are zero at $t=t_{0}$ and at $t=t_{0}+T / 2$ but are not simultaneously zero at any value of $t \in\left(t_{0}, t_{0}+T / 2\right)$, then $\varphi(t)$ is a $S_{1}-$ symmetric periodic solution of period $T$.

Equations (4) are also invariant under the symmetry

$$
\left(t, x_{1}, \dot{x}_{1}, x_{2}, \dot{x}_{2}\right) \longrightarrow\left(-t,-x_{1}, \dot{x}_{1}, x_{2},-\dot{x}_{2}\right),
$$

i.e., the symmetry with respect to the $x_{2}$-axis, which will be denoted by $S_{2}$ and is characterized as follows. 
Proposition 3. Let $\varphi(t)=\left(x_{1}(t), \dot{x}_{1}(t), x_{2}(t), \dot{x}_{2}(t)\right)$ be a solution of system (4). If $x_{1}(t)$ and $\dot{x}_{2}(t)$ are zero at $t=t_{0}$ and at $t=t_{0}+T / 2$ but are not simultaneously zero at any value of $t \in\left(t_{0}, t_{0}+T / 2\right)$, then $\varphi(t)$ is a $S_{2}-$ symmetric periodic solution of period $T$.

We note that there could be periodic solutions of (4) that are simultaneously $S_{1}$ - and $S_{2}$-symmetric. These periodic solutions will be called doubly symmetric periodic solutions and are characterized by the following result.

Proposition 4. Let $\varphi(t)=\left(x_{1}(t), \dot{x}_{1}(t), x_{2}(t), \dot{x}_{2}(t)\right)$ be a solution of system (4).

(a) If $\dot{x}_{1}(t)$ and $x_{2}(t)$ are zero at $t=t_{0}$ and $x_{1}(t)$ and $\dot{x}_{2}(t)$ are zero at $t=t_{0}+T / 4$ but are not simultaneously zero at any value of $t \in\left(t_{0}, t_{0}+T / 4\right)$, then $\varphi(t)$ is a doubly symmetric periodic solution of period $T$.

(b) If $x_{1}(t)$ and $\dot{x}_{2}(t)$ are zero at $t=t_{0}$ and $\dot{x}_{1}(t)$ and $x_{2}(t)$ are zero at $t=t_{0}+T / 4$ but are not simultaneously zero at any value of $t \in\left(t_{0}, t_{0}+T / 4\right)$, then $\varphi(t)$ is a doubly symmetric periodic solution of period $T$.

For the proof of the above propositions, see for instance [3].

\section{The Continuation of SOME SYMmetric ElLiPtiC ORBits}

Poincaré defines periodic orbits of the second kind as those which are generated from elliptic 2-body orbits. Again for more details see [10].

To prove the continuation of the circular and symmetric elliptic orbits we change the coordinates from the polar coordinates $(r, \theta, R, \Theta)$ to Delaunay's coordinates $(l, g, L, G)$. We recall here the geometrical meaning of these coordinates

- $l$ is the mean anomaly and is measured from the perihelion;

- $g$ is the argument of the pericenter and determines the orientation of the major semi-axis of the ellipse in the plane of motion;

- $L$ is related to the major semi-axis, $a$, by $L=a^{1 / 2}$;

- $G$ is related to the major semi-axis, $a$, and to the eccentricity, $e$, by $G=\left[a\left(1-e^{2}\right)\right]^{1 / 2}$.

In Delaunay coordinates the Hamiltonian (3) takes the form

$$
H=-\frac{1}{2 L^{2}}-G+O\left(\mu^{2}\right)
$$

Following [11], we can prove in a similar way to the circular planar restricted 3-body problem, the continuation to $\mu>0$ small of the elliptic orbits for the collision restricted 3 -body problem. Taking into account the 
possible symmetries of the periodic solutions we have three different situations.

5.1. Continuation of $S_{1}$-symmetric periodic orbits. We start to remark that we have 8 different $S_{1}$-symmetric elliptic orbits for the Kepler problem in rotating coordinates which can be continued into $S_{1}$-symmetric periodic orbits of the collision restricted 3 -body problem when $\mu>0$ is small. These different orbits are easy to describe in terms of the initial conditions in Delaunay coordinates $g, l$. For the counter-clockwise motion of the infinitesimal mass we can have the following initial conditions

(a) $g(0)=0, l(0)=0$, which means that the pericenter is on the positive $x_{1}$-semiaxis and the infinitesimal mass is at the pericenter;

(b) $g(0)=\pi, l(0)=0$ which means that the pericenter is on the negative $x_{1}$-semiaxis and the infinitesimal mass is at the pericenter;

(c) $g(0)=0, l(0)=\pi$ which means that the pericenter is on the positive $x_{1}$-semiaxis and the infinitesimal mass is at the apocenter;

(d) $g(0)=\pi, l(0)=\pi$ which means that the pericenter is on the negative $x_{1}$-semiaxis and the infinitesimal mass is at the apocenter.

There are other 4 different $S_{1}$-symmetric elliptic orbits for the clockwise motion of the infinitesimal mass.

Now we analyze the situation when the motion of the infinitesimal body is counter-clockwise. In Delaunay coordinates $(l, g, L, G)$, an orthogonal crossing of the $x_{1}$-axis at a time $t_{0}$ is described by

$$
l\left(t_{0}\right)=n_{1} \pi, \quad g\left(t_{0}\right)=n_{2} \pi, \quad n_{1}, n_{2} \text { integers. }
$$

In order to have a $S_{1}$-symmetric orbit we need to have 2 different orthogonal crossings of the $x_{1}$-axis. We will prove that, $\bmod 2 \pi$ in time, the $4 S_{1}-$ symmetric elliptic orbits can be continued into 16 different $S_{1}$-symmetric orbits of the collision restricted 3-body problem when $\mu$ is small. These 16 possibilities come from the fact that we can have the first orthogonal crossings of the $x_{1}$-axis in any of the situation $(a),(b),(c)$ or $(d)$ and the second orthogonal crossings of the $x_{1}$-axis also in any of this 4 different situations.

In the situation when the motion of the infinitesimal body is clockwise we will obtain other 16 different $S_{1}$-symmetric periodic orbits of the collision restricted 3-body problem.

Theorem 5. Let $p, q$ be relatively prime integers and $T=2 \pi p / q$. Then the elliptic T-periodic orbit of the Kepler problem in rotating coordinates which satisfies

$$
l(0)=n_{1} \pi, \quad g(0)=n_{2} \pi, \quad L^{3}(0)=p / q, \quad G(0)=\text { constant }
$$


can be continued into a $S_{1}$-symmetric periodic of period $2 \pi p$ orbit of the collision restricted 3-body problem for small values of $\mu>0$.

Proof. The Hamiltonian of the collision restricted 3-body problem in Delaunay coordinates for small $\mu$ is given by (5) and the equations of motion are

$$
\begin{array}{ll}
\dot{i}=\frac{1}{L^{3}}+O\left(\mu^{2}\right), & \dot{L}=0+O\left(\mu^{2}\right), \\
\dot{g}=-1+O\left(\mu^{2}\right), & \dot{G}=0+O\left(\mu^{2}\right) .
\end{array}
$$

For $\mu=0$ we consider the elliptic orbit satisfying the initial conditions (7). The motion on this ellipse is

$$
\begin{aligned}
& l=t q / p+n_{1} \pi, \quad L=(p / q)^{1 / 3}, \\
& g=-t+n_{2} \pi, \quad G=\text { constant. }
\end{aligned}
$$

The period of the infinitesimal mass following this ellipse is $T=2 \pi L^{3}=$ $2 \pi p / q$. Thus, the period of the motion of the three body together is $T^{\prime}=$ $2 \pi p$, so $g\left(T^{\prime} / 2\right)=\left(-p+n_{2}\right) \pi$ and $l\left(T^{\prime} / 2\right)=\left(q+n_{1}\right) \pi$. Hence, that orbit satisfies (6) (and is thus $S_{1}$-symmetric).

Now for small $\mu>0$ we consider solutions satisfying (8) and the initial conditions

$$
l(0)=n_{1} \pi, \quad g(0)=n_{2} \pi, \quad L(0)=L_{0}, \quad G(0)=G_{0} .
$$

We wish to solve

$$
\begin{aligned}
& \psi_{1}\left(L_{0}, G_{0}, t, \mu\right)=0, \\
& \psi_{2}\left(L_{0}, G_{0}, t, \mu\right)=0,
\end{aligned}
$$

for $L_{0}$ near $(p / q)^{1 / 3}$ and $t$ near $T^{\prime} / 2$ where

$$
\psi_{1}\left(L_{0}, G_{0}, t, \mu\right)=g(t)+\left(p-n_{2}\right) \pi,
$$

and

$$
\psi_{2}\left(L_{0}, G_{0}, t, \mu\right)=l(t)-\left(q+n_{1}\right) \pi .
$$

By the Implicit Function Theorem, it suffices to show that

$$
\operatorname{det}\left(\begin{array}{cc}
\partial \psi_{1} / \partial t & \partial \psi_{2} / \partial t \\
\partial \psi_{1} / \partial L_{0} & \partial \psi_{2} / \partial L_{0}
\end{array}\right) \neq 0
$$

at the elliptic orbit, but for $\mu=0$ we have

$$
\operatorname{det}\left(\begin{array}{cc}
\partial g / \partial t & \partial l / \partial t \\
\partial g / \partial L_{0} & \partial l / \partial L_{0}
\end{array}\right)=\operatorname{det}\left(\begin{array}{cc}
-1 & q / p \\
0 & -3 t(q / p)^{4 / 3}
\end{array}\right) \neq 0
$$

for any $t>0$. 
We want to remark that some of these different families of $S_{1}$-symmetric periodic orbits are doubly symmetric but we will consider these situations later on.

5.2. Continuation of $S_{2}$-symmetric periodic orbits. Like in previous subsection we have 8 different $S_{2}$-symmetric elliptic orbits for the Kepler problem in rotating coordinates which can be continued into the $S_{2}-$ symmetric orbits of the collision restricted 3-body problem when $\mu$ is small. Again, these different orbits are easy to describe in terms of the initial conditions in Delaunay coordinates $g, l$. For the counter-clockwise motion of the infinitesimal mass we can have the following initial conditions

(a) $g(0)=-\pi / 2, l(0)=0$, which means that the pericenter is on the negative $x_{2}$-semiaxis and the infinitesimal mass is at the pericenter;

(b) $g(0)=-\pi / 2, l(0)=\pi$ which means that the pericenter is on the negative $x_{2}$-semiaxis and the infinitesimal mass is at the apocenter;

(c) $g(0)=\pi / 2, l(0)=\pi$ which means that the pericenter is on the positive $x_{2}$-semiaxis and the infinitesimal mass is at the apocenter;

(d) $g(0)=\pi / 2, l(0)=0$ which means that the pericenter is on the positive $x_{2}$-semiaxis and the infinitesimal mass is at the pericenter.

There are other 4 different $S_{1}$-symmetric elliptic orbits for the clockwise motion of the infinitesimal mass.

We start to analyze the situation when the motion of the infinitesimal body is counter-clockwise. In Delaunay coordinates $(l, g, L, G)$, an orthogonal crossing of the $x_{2}$-axis at a time $t_{0}$ is described by

$$
l\left(t_{0}\right)=n_{1} \pi, \quad g\left(t_{0}\right)=n_{2} \pi+\pi / 2, \quad n_{1}, n_{2} \text { integers. }
$$

and to have an $S_{2}$-symmetric orbit we need to have 2 different orthogonal crossings of the $x_{2}$-axis. We will see that, mod $2 \pi$ in time, the 4 elliptic orbits can be continued into 16 different $S_{2}$-symmetric periodic orbits of the collision restricted 3 -body problem when $\mu$ is small. These 16 possibilities come from the fact that we can have the first orthogonal crossings of the $x_{2}$-axis in any of the situation $(a),(b),(c)$ or $(d)$ and the second orthogonal crossings of the $x_{2}$-axis also in any of this 4 different situations.

In the situation when the motion of the infinitesimal body is clockwise we will obtain other 16 different $S_{2}$-symmetric orbits of the collision restricted 3-body problem.

Theorem 6. Let $p, q$ be integers and $T=2 \pi p / q$. Then the elliptic $T-$ periodic orbit of the Kepler problem in rotating coordinates which satisfies

$$
l(0)=n_{1} \pi, \quad g(0)=n_{2} \pi+\pi / 2, \quad L^{3}(0)=p / q, \quad G(0)=\text { constant }
$$


can be continued into a $S_{2}$-symmetric periodic orbit of period $2 \pi p$ of the collision restricted 3-body problem for small values of $\mu>0$.

Proof. Like in the proof of Theorem 5, for $\mu=0$ we consider the elliptic orbit satisfying the initial conditions (10). The motion on this ellipse is,

$$
\begin{array}{r}
l=t q / p+n_{1} \pi, \quad L=(p / q)^{1 / 3}, \\
g=-t+n_{2} \pi+\pi / 2, \quad G=\text { constant. }
\end{array}
$$

The period of the infinitesimal mass following this ellipse is $T=2 \pi L^{3}=$ $2 \pi p / q$. Thus, the period of the motion of the three bodies together is $T^{\prime}=2 \pi p$, so $g\left(T^{\prime} / 2\right)=\left(-p+n_{2}\right) \pi+\pi / 2$ and $l\left(T^{\prime} / 2\right)=\left(n_{1}+q\right) \pi$. Hence that orbit satisfies (9) (and is thus $S_{2}$-symmetric).

Now for small $\mu>0$ we consider solutions satisfying (8) and the initial conditions

$$
l(0)=n_{1} \pi, \quad g(0)=n_{2} \pi+\pi / 2, \quad L(0)=L_{0}, \quad G(0)=G_{0} .
$$

We wish to solve

$$
\begin{aligned}
& \psi_{1}\left(L_{0}, G_{0}, t, \mu\right)=0, \\
& \psi_{2}\left(L_{0}, G_{0}, t, \mu\right)=0,
\end{aligned}
$$

for $L_{0}$ near $(p / q)^{1 / 3}$ and $t$ near $T^{\prime} / 2$ where

$$
\psi_{1}\left(L_{0}, G_{0}, t, \mu\right)=g(t)+\left(p-n_{2}\right) \pi-\pi / 2,
$$

and

$$
\psi_{2}\left(L_{0}, G_{0}, t, \mu\right)=l(t)-\left(n_{1}+q\right) \pi .
$$

The rest of the proof is the same as in the proof of Theorem 5 .

5.3. Continuation of doubly symmetric periodic orbits. We saw in the previous section, in Proposition 4, the characterization of the doubly symmetric periodic orbit of (4). Taking into account (6) and (9) this characterization implies the following conditions in Delaunay coordinates

$$
l\left(t_{0}\right)=n_{1} \pi, \quad g\left(t_{0}\right)=n_{2} \pi, \quad n_{1}, n_{2} \text { integers },
$$

(orthogonal crossing on the $x_{1}$-axis) and

$$
l\left(t_{0}+T / 4\right)=n_{3} \pi, \quad g\left(t_{0}+T / 4\right)=n_{4} \pi+\pi / 2, \quad n_{3}, n_{4} \text { integers, }
$$

(orthogonal crossing on the $x_{2}$-axis).

Now counting all the possible relative positions of these orthogonal crossings of $x_{1}$-axis and $x_{2}$-axis, is easy to see that, mod $2 \pi$ in time, we can obtain 16 different doubly symmetric periodic orbits of the collision restricted 3 -body problem. Note that we have 16 orbits rotating in counter-clockwise 
and 16 orbits rotating in clockwise. Next theorem will show that the doubly symmetric elliptic orbits of the Kepler problem can be continued into doubly symmetric periodic orbits of the collision restricted 3-body problem when $\mu>0$ is small.

Theorem 7. Let $p$ be an odd integer and $q$ be an even integer and $T=$ $2 \pi p / q$. Then the elliptic $T$-periodic orbit of the Kepler problem in rotating coordinates which satisfies the initial conditions

$$
l(0)=n_{1} \pi, \quad g(0)=n_{2} \pi, \quad L^{3}(0)=p / q, \quad G(0)=\text { constant },
$$

can be continued into a doubly symmetric periodic orbit of the collision restricted 3-body problem for small values of $\mu>0$.

Proof. Like in the proof of Theorem 5, for $\mu=0$ we consider the elliptic orbit satisfying the initial conditions (13). The motion on this ellipse is,

$$
\begin{aligned}
& l=t q / p+n_{1} \pi, \quad L=(p / q)^{1 / 3}, \\
& g=-t+n_{2} \pi, \quad G=\text { constant. }
\end{aligned}
$$

The period of the infinitesimal mass following this ellipse is $T=2 \pi L^{3}=$ $2 \pi p / q$. Thus, the period of the motion of the three bodies together is $T^{\prime}=2 \pi p$, so $g\left(T^{\prime} / 4\right)=-\pi p / 2+n_{2} \pi$ and $l\left(T^{\prime} / 4\right)=\pi q / 2+n_{1} \pi$. Taking into account that $p$ is odd and $q$ is even, the orbit satisfies (12) (and is thus doubly symmetric).

Now for small $\mu>0$ we consider solutions satisfying (8) and the initial conditions

$$
l(0)=n_{1} \pi, \quad g(0)=n_{2} \pi, \quad L(0)=L_{0}, \quad G(0)=G_{0} .
$$

We wish to solve

$$
\begin{aligned}
& \psi_{1}\left(L_{0}, G_{0}, t, \mu\right)=0, \\
& \psi_{2}\left(L_{0}, G_{0}, t, \mu\right)=0,
\end{aligned}
$$

for $L_{0}$ near $(p / q)^{1 / 3}$ and $t$ near $T^{\prime} / 4$ where

$$
\psi_{1}\left(L_{0}, G_{0}, t, \mu\right)=g(t)+\pi p / 2-n_{2} \pi,
$$

and

$$
\psi_{2}\left(L_{0}, G_{0}, t, \mu\right)=l(t)-\pi q / 2-n_{1} \pi .
$$

The rest of the proof is the same as in the proof of Theorem 5 .

We want to mention that the initial conditions (13) in the previous theorem have been chosen such that the orbit start with an orthogonal crossing with the $x_{1}$-axis. We can prove a similar theorem in the case when we choose the initial conditions such that the orbit will start with an orthogonal crossing with the $x_{2}$-axis. We note that also in this case we can continue 
the elliptic periodic symmetric orbits just in the situation when $p$ is odd and $q$ is even.

\section{The COntinuation of COMEts orbits}

Following Section E of Chapter VI of [7], we will consider the situation when the infinitesimal body is far from the primaries in which case it will be called the comet. To consider orbits close to infinity, scale the variables by $x \rightarrow \varepsilon^{-2} x, y \rightarrow \varepsilon y$. This change is symplectic with multiplier $\varepsilon$. Thus, with $H \rightarrow \varepsilon H$, since $\varepsilon$ is a small parameter, the Hamiltonian (2) becomes

$$
H=-x^{T} K y+\varepsilon^{3}\left\{\frac{\|y\|^{2}}{2}-\frac{1}{\|x\|}\right\}+O\left(\varepsilon^{7}\right) .
$$

Changing to polar coordinates we get

$$
H=-\Theta+\varepsilon^{3}\left[\frac{1}{2}\left(R^{2}+\frac{\Theta^{2}}{r^{2}}\right)-\frac{1}{r}\right]+O\left(\varepsilon^{7}\right),
$$

and the equations of motion are

$$
\begin{array}{rrr}
\dot{r} & =\varepsilon^{3} R, & \dot{R}=\varepsilon^{3}\left(\frac{\Theta^{2}}{r^{3}}-\frac{1}{r^{2}}\right), \\
\dot{\theta}=-1+\varepsilon^{3} \frac{\Theta}{r^{2}}, & \dot{\Theta}=0 .
\end{array}
$$

The terms of order $\varepsilon^{7}$ have been dropped from the equations in (17), and to this order of approximation, $\Theta$ is an integral.

Theorem 8. There exist 2 one-parameter families of nearly circular large elliptic periodic solutions of the collision restricted 3-body problem for all values of the parameter $\mu$. These orbits tend to infinity.

Proof. A pair of circular periodic solutions of (17) are $\Theta= \pm c, R=0$, $r=c^{2}$, where $c$ is a constant. The period of these solutions is $2 \pi c^{3} /\left(c^{3}-\varepsilon^{3}\right)$. Linearizing the $r$ and $R$ equations about these solutions gives

$$
\dot{r}=\varepsilon^{3} R, \quad \dot{R}=-\frac{\varepsilon^{3}}{c^{6}} r .
$$

Equations (18) have solutions of the form $\exp \left( \pm i \varepsilon^{3} t / c^{3}\right)$, and so the nontrivial multipliers of the circular orbits of (17) are $\exp \left( \pm i \varepsilon^{3} 2 \pi /\left(c^{3}-\varepsilon^{3}\right)\right)=$ $1 \pm 2 \pi i \varepsilon^{3} / c^{3}+O\left(\varepsilon^{6}\right)$.

Consider the period map in a level surface of the Hamiltonian about this circular orbit. Let $u$ be the coordinate in this surface, with $u=0$, corresponding to the circular orbit when $\varepsilon=0$. The period map has a fixed point at the origin up to terms of order $\varepsilon^{3}$ and is the identity up to terms of order $\varepsilon^{2}$, and at $\varepsilon^{3}$ there is a term whose Jacobian has eigenvalues $\pm 2 \pi i / c^{3}$. That is, the period map is of the form $P(u)=u+\varepsilon^{3} p(u)+O\left(\varepsilon^{6}\right)$, where 
$p(0)=0$, and $\partial p(0) / \partial u$ has eigenvalues $\pm 2 \pi i / c^{3}$; so, in particular, $\partial p(0) / \partial u$ is nonsingular. Apply the implicit function theorem to $G(u, \varepsilon)=(P(u)-$ $u) / \varepsilon^{3}=p(u)+O\left(\varepsilon^{3}\right)$. Since $G(0,0)=0$ and $\partial G(0,0) / \partial u=\partial p(0) / \partial u$, there is a smooth function $\bar{u}(\varepsilon)$ such that $G(\bar{u}(\varepsilon), \varepsilon)=0$ for all $\varepsilon$ sufficiently small. Thus, the two solutions can be continued from the equations in (17) to the full equations, where the $O\left(\varepsilon^{7}\right)$ terms are included. In the scaled variables, these solutions have period $T \simeq 2 \pi$.

We note that the two families mentioned in the statement of Theorem 8 correspond to counter-clockwise and clockwise motion.

\section{Appendix}

We recall some definitions and results about the continuation of periodic solutions for periodic differential equations. For more details see [6] or [7].

Consider a periodic system of equations of the form $\dot{x}=f(t, x, v)$ where $v$ is a parameter, and $f$ is $T$-periodic in $t, f(t+T, x, v)=f(t, x, v)$. Let $\phi(t, \xi, v)$ be the general solution, $\phi(0, \xi, v)=\xi$.

A necessary and sufficient condition for $\phi\left(t, \xi^{\prime}, v^{\prime}\right)$ to be periodic with a period $T$ is $\phi\left(T, \xi^{\prime}, v^{\prime}\right)=\xi^{\prime}$.

A $T$-periodic solution $\phi\left(t, \xi^{\prime}, v^{\prime}\right)$ can be continued if there is a smooth function $\bar{\xi}(v)$ such that $\bar{\xi}\left(v^{\prime}\right)=\xi^{\prime}$, and $\phi\left(T, \bar{\xi}\left(v^{\prime}\right), v^{\prime}\right)$ is $T$-periodic.

The multipliers of the $T$-periodic solution $\phi\left(t, \xi^{\prime}, v^{\prime}\right)$ are the eigenvalues of the $\partial \phi\left(T, \xi^{\prime}, v^{\prime}\right) / \partial \xi$.

A $T$-periodic solution can be continued if all of its multipliers are different from +1 .

\section{ACKNOWLEDGEMENTS}

The second author thanks Centre de Recerca Matemàtica for the hospitality and facilities for doing this work.

\section{REFERENCES}

[1] M. Corbera, J. Llibre, Families of periodic orbits for the spatial isosceles 3-body. SIAM J. Math. Anal. 35 (2004), 1311-1346.

[2] M. Corbera, J. Llibre, Periodic orbits of a collinear restricted three body problem, Celestial Mech. Dynam. Astronom. 86 (2003), 163-183.

[3] M. Corbera, J. Llibre, Periodic orbits of the Sitnikov problem via a Poincaré map, Celestial Mech. Dynam. Astronom. 77 (2000), 273-303. 
[4] M. Corbera, J. Llibre, On symmetric periodic orbits of the elliptic Sitnikov problem via the analytic continuation method. Celestial mechanics (Evanston, IL, 1999), 91127, Contemp. Math. 292 Amer. Math. Soc., Providence, RI, 2002.

[5] M. Corbera, J. Llibre, Periodic and quasi-periodic orbits for the spatial isosceles three-body problem. Third Conference on Celestial Mechanics (Spanish) (Valladolid, 2000), Monogr. Semin. Mat. García Galdeano, 21 (2001), 37-44, Univ. Zaragoza, Zaragoza.

[6] K.R. Meyer, Periodic Solutions of the $N$-Body Problem, Lecture Notes in Math. 1719, Springer-Verlag, Berlin, 1999.

[7] K.R. Meyer, G.R. Hall, Introduction to Hamiltonian Dynamical Systems and the N-Body Problem, AMS 90, Springer-Verlag, 1992.

[8] H. Poincaré, Les Mèthodes Nouvelles de la Méchanique Céleste, 3 Vols., GauthierVillar, Paris, 1892-1899, (reprinted by Dover, New York, 1957).

[9] A. Roy, Orbital motions, Adam Hilger Ltd, Bristol, England, 1978.

[10] V. Szebehely, Theory of orbits, Academic Press, New York, 1967.

[11] S. Sternberg, Celestial Mechanics, W.A. Bemjamin, Inc., New York, Amsterdam, 1969.

1 Departament de Matemàtiques Universitat Autònoma de Barcelona, 08193

Bellaterra,, Barcelona, Spain

E-mail address: jllibre@mat.uab.es

2 Department of Mathematics and Informatics, University of Oradea, UniVersity Street 1, 410087 Oradea, Romania

E-mail address: dpasca@uoradea.ro

3 Current address: Centre de Recerca Matemàtica, 08193 Bellaterra, BARCELONA, Spain

E-mail address: dpasca@crm.es 